\title{
DOENÇA DE CHAGAS E PATENTES FARMACÊUTICAS: PESQUISA EXPLORATÓRIA SOBRE A EFETIVIDADE DO SISTEMA DE PATENTES
}

\author{
MARCOS VINÍ́CIO CHEIN FERES ${ }^{\dagger}$ \\ LORENA ABBAS DA SILVA ${ }^{+\dagger}$
}

Resumo: A presente pesquisa visa indagar se o sistema jurídico de patentes é efetivo o suficiente, no que diz respeito à sua aspiração moral, para incentivar o desenvolvimento de produtos de saúde, tendo por foco a análise dos depósitos de patentes para a doença de Chagas. A análise metodológica utilizada consiste numa abordagem qualitativa dos dados coletados de fontes diretas e indiretas, dentre as quais o site do Instituto Nacional da Propriedade Industrial. A partir dos dados coletados foram construídas três tabelas, as quais se referem ao número de depósitos relacionados à doença, o número de depósitos por depositante e o perfil funcional dos depósitos. Este estudo se baseia no marco teórico da luta pelo reconhecimento, de Axel Honneth, conjugado com o viver plenamente a lei, de Zenon Bankowski. A partir dos dados coletados relativos a doença de Chagas, infere-se a pouca efetividade do sistema jurídico de patentes com relação ao incentivo à inovação voltada para interesses sociais locais. Os interesses de mercado se sobrepõem à promessa vaga do sistema jurídico de patentes concernente à busca pelo desenvolvimento científico e tecnológico ancorado nos interesses sociais.

Palavras-Chave: Propriedade intelectual; Patentes; Doença de Chagas; Doenças negligenciadas; Pesquisa empírica.

\footnotetext{
+ Doutor em Direito Econômico Professor Associado da UFJF. Professor do Programa de Pós-Graduação Estrito Senso em Direito e Inovação. Pesquisador de Produtividade PQ2 CNPq. Apoiado financeiramente pela FAPEMIG.

+ + Bolsista DS/CAPES. Mestranda em Direito e Inovação na Faculdade de Direito da UFJF. Bolsista do PROBIC/FAPEMIG 2016/2017.
} 
ABSTRACT: This research aims to question if the patent system is effective enough, as far as its morality of aspiration is concerned, to incentivize the development of health products, considering the analysis of patent applications referred to Chagas disease. The methodological analysis utilized consists in the qualitative approach of collected data from both direct and indirect sources, including the website of National Institute of Industrial Property (INPI). Stemming from the collected data, three tables are elaborated, concerning the number of patent applications, the number of applications by depositors and the role of the patent applications. This study is based on the theoretical reference of struggle for recognition, from Axel Honneth, correlated with Zenon Bankowski's legal theory of living lawfully. Considering the Chagas diseases patent applications, it is possible to infer that the patent system is not effective enough to fulfill the aspiration of scientific and technological development socially oriented. The market interests overcome the vague promise of the patent system concerning the constitutional purpose of scientific and technological development intertwined with social welfare.

KEYWORDS: Intellectual property; Patent; Neglected diseases; Empirical research. 


\section{INTRODUÇÃO}

A doença de Chagas, também conhecida como tripanossomíase americana, é causada pelo parasita protozoário Trypanosoma cruzi e foi originariamente descoberta na cidade de Lassance, localizada no interior do estado de Minas Gerais ${ }^{1}$. Foi o pesquisador Carlos Justiniano Ribeiro Chagas o responsável pelo primeiro relato científico sobre a doença, que mais tarde viria a levar o seu nome, ao realizar uma descrição minuciosa sobre o parasita, o seu ciclo evolutivo, o vetor barbeiro e o hospedeiro doméstico da doença, o gato ${ }^{2}$.

Entre as diversas formas de infecção é possível destacar a transfusão de sangue, transmissão oral por alimentos contaminados, transmissão vertical via placenta (da genitora para o filho) e transplante de órgãos. Todavia, o modo mais recorrente de contaminação se dá pelo contato com as fezes do inseto popularmente conhecido como barbeiro, da família Triatominae. A picada do inseto provoca coceira e, com isso, o tripanossomo facilmente penetra no organismo pela mucosa dos olhos, nariz e boca, além da pele do paciente ${ }^{3}$.

Apesar de ser endêmica na região das Américas, predominantemente na América Latina, nos últimos anos a doença de Chagas tem sido detectada em outras regiões do globo, como no Canadá e nos Estados Unidos, e em países da Europa, por causa da alta mobilidade populacional e da grande quantidade de transfusões sanguíneas ocorridas entre infectados e pessoas desses países ${ }^{4}$.

1 MALAFAIA, Guilherme; RODRIGUES, Aline Sueli de Lima. Centenário do descobrimento da doença de Chagas: desafios e perspectivas. [Editorial]. Revista da Sociedade Brasileira de Medicina Tropical. Uberaba, v. 43, n. 5, p. 483-485, set-out. 2010. Disponível em: $<$ http://www.scielo.br/scielo.php?script=sci arttext\&pid=S003786822010000500001>. Acesso em: 15 mar. 2016.

2 FUNDAÇÃO OSWALDO CRUZ (FIOCRUZ) (Invivo). História: Carlos Chagas. Publicado em 07 jan. 2003. Disponível em: <http://www.invivo.fiocruz.br/cgi/cgilua.exe/sys/start.htm?infoid=109\&sid=7>.

Acesso em: 10 jun. 2016.

3 ORGANIZAÇÃO MUNDIAL DA SAÚDE (OMS). Trabalhando para Superar o Impacto Global de Doenças Tropicais Negligenciadas: Primeiro relatório da OMS sobre Doenças Tropicais Negligenciadas 2010, p. 75. Disponível em: <http://www.who.int/eportuguese/publications/pt/>. Acesso em: 15 mar. 2016.

${ }^{4}$ Cf. ORGANIZAÇÃO MUNDIAL DA SAÚDE (OMS). Trabalhando para Superar o

Impacto Global de Doenças Tropicais Negligenciadas: Primeiro relatório da OMS 
Não obstante a disseminação de tal doença em outros espaços, o mal de Chagas ainda figura no quadro de doenças consideradas negligenciadas, que são patologias causadas geralmente por agentes infecciosos e parasitários e não despertam interesse da indústria farmacêutica diante do público-alvo de baixo poder aquisitivo ${ }^{5}$. O impacto do aspecto econômico fica ainda mais evidente na análise realizada por Philipsborn e outros ${ }^{6}$ sobre as doenças que acometem os países mais pobres. Os autores afirmam que, embora sejam responsáveis por cerca de $13.8 \%$ da carga global de danos, as enfermidades negligenciadas recebem apenas $1.34 \%$ dos investimentos em pesquisa e desenvolvimento em saúde ${ }^{7}$.

Segundo relatório ${ }^{8}$ da iniciativa Medicamentos para Doenças Negligenciadas, DNDi, estima-se que existem cerca de 8 milhões de pessoas na América Latina ${ }^{9}$ com doença de Chagas e que, anualmente, menos de $1 \%$ dessas pessoas recebem tratamento.

Em um plano correlato, o instituto jurídico utilizado para incentivar o surgimento e desenvolvimento de medicamentos é o direito à patente,

sobre Doenças Tropicais Negligenciadas 2010, p. 75. Disponível em: <http://www.who.int/eportuguese/publications/pt/>. Acesso em: 15 mar. 2016.

${ }_{5}^{5}$ SOUZA, Wanderley de (Org.). Doenças Negligenciadas. Rio de Janeiro, RJ: Academia Brasileira de Ciências, 2010. Disponível em: <http://www.abc.org.br/IMG/pdf/doc199.pdf>. Acesso em: 15 mar. 2016.

6 PHILIPSBORN, Peter von. STEINBEIS, Fridolin. BENDER, Max E. REGMI, Sadie Regmi. TINNEMANN, Peter. Poverty-related and neglected diseases - an economic and epidemiological analysis of poverty relatedness and neglect in research and development. Global Health Action, 2015, pp. 2-4. DOI: <10.3402/gha.v8.25818>. Acesso em: 15 jun. 2018.

${ }^{7}$ Cf. PHILIPSBORN, Peter von. STEINBEIS, Fridolin. BENDER, Max E. REGMI, Sadie Regmi. TINNEMANN, Peter. Poverty-related and neglected diseases - an economic and epidemiological analysis of poverty relatedness and neglect in research and development. Global Health Action, 2015, pp. 2-4. DOI: <10.3402/gha.v8.25818>. Acesso em: 15 jun. 2018.

8 INICIATIVA MEDICAMENTOS PARA DOENÇAS NEGLIGENCIADAS (DNDi) (América Latina). Relatório Anual DNDi América Latina 2012-2013, p. 16. Disponível em: $\quad<$ http://www.dndial.org/pt/centro-de-documentacao/relatorio-anual.html $>$. Acesso em: 20 mar. 2016.

9 Peter Hotez e Ricardo T. Fujiwara apresentam estimativa semelhante em Brazil's neglected tropical diseases: An overview and a report card. Microbes And Infection 16, 2014, p. 604. Disponível em: <http://www.ncbi.nlm.nih.gov/pubmed/25088506?dopt=Abstract $>$. Acesso em: 14 jun. 2018. 
com a concessão de exploração econômica exclusiva, em dado lapso de tempo, ao seu criador ${ }^{10}$.

Entretanto, no que se refere às chamadas doenças negligenciadas, verifica-se o desinteresse na pesquisa e elaboração de medicamentos para tais doenças, sobretudo pela ausência de retorno financeiro às indústrias privadas ${ }^{11}$. O propósito dessa pesquisa é o de questionar se o sistema jurídico de patentes possui efetividade, no que diz respeito à sua aspiração moral, para o desenvolvimento de produtos de saúde, tendo por foco a análise dos depósitos de patentes para a doença de Chagas. O plano teórico utilizado para a realização dessa pesquisa é o da luta pelo reconhecimento, em Axel Honneth ${ }^{12}$, e do viver plenamente o direito, em Zenon Bankowski ${ }^{13}$.

Em um primeiro momento, são elucidados os referenciais teóricos e a metodologia utilizada, bem como o procedimento de coleta de dados. Posteriormente, os dados obtidos são interpretados. E, por fim, os resultados são discutidos com a demonstração final da inferência descritiva alcançada no processo de pesquisa.

\section{Metodologia E Plano teóRico}

Antes de apresentar os dados coletados e proceder à realização da inferência, cumpre esclarecer neste tópico o quadro teórico e a metodologia utilizada para alcançar os resultados do trabalho.

\section{O referencial teórico}

O substrato teórico aplicado para o desenvolvimento desta pesquisa surge da adoção conjunta das teorias da luta pelo reconhecimento de Axel

\footnotetext{
${ }^{10}$ BARBOSA, Denis Borges. Uma Introdução à Propriedade Intelectual. $2^{\underline{a}}$ ed. Rio de Janeiro: Lúmen Júris, 2003.

${ }^{11}$ Cf. INICIATIVA MEDICAMENTOS PARA DOENÇAS NEGLIGENCIADAS (DNDi) (América Latina). Relatório Anual DNDi América Latina 2012-2013, p. 17. Disponível em: $\quad$ http://www.dndial.org/pt/centro-de-documentacao/relatorio-anual.html>. Acesso em: 20 mar. 2016.

12 HONNETH, Axel. Luta por reconhecimento: a gramática moral dos conflitos sociais. Tradução: Luiz Repa. Apresentação: Marcos Nobre. São Paulo: Editora 34, 2003, 296 p. ${ }^{13}$ BANKOWSKI, Zenon. Vivendo Plenamente a Lei: a Lei do Amor e o Amor pela Lei. Trad. Lucas Dutra Bertolozzo, Luiz Reimer Rodrigues Rieffel e Arthur Maria Ferreira Neto. Rio de Janeiro, RJ: Elsevier Campus, 2008, 289 p.
} 
Honneth ${ }^{14}$ e do viver plenamente a lei de Zenon Bankowski ${ }^{15}$. Juntas, estas teorias norteiam este estudo com vistas a uma investigação que transcenda a frieza da letra da lei e dos números, proporcionando um plano teórico consistente para que os fenômenos evidenciados sejam analisados com um olhar mais dinâmico e interdisciplinar, sobretudo visando ao bem comum e à máxima realização dos direitos garantidos a todos os cidadãos.

Em Honneth ${ }^{16}$, o processo de luta pelo reconhecimento consagra o papel do indivíduo encaixado no seu respectivo locus social, de forma que a construção da identidade individual só é possível numa perspectiva intersubjetiva, quer dizer, nos processos de interação e relação social. Para o autor, esse processo ocorre em três fases correlatas: do amor, do direito e da estima social.

Como numa gradação, essas etapas se complementam, a partir de seus respectivos graus de colaboração na formação da identidade. A primeira etapa, do amor, é a fase em que o indivíduo articula suas carências subjetivas visando à construção da autoconfiança. $\mathrm{O}$ indivíduo começa a se compreender como ser único, cujas características imanentes o diferencia dos demais membros da comunidade. Na fase do direito, esta individualidade é contraposta com os demais sujeitos integrantes do corpo social, de modo a criar limites e regular a vivência, na construção do autorrespeito. Por fim, na fase da estima social, o indivíduo corrobora sua individualidade na construção da solidariedade, participando como membro e se sentindo socialmente estimado no ambiente que integra. A noção de bem-estar é vista como uma construção dialética, que parte do indivíduo para a coletividade e da coletividade para o indivíduo ${ }^{17}$.

O processo de construção da identidade necessita de respaldo das normas construídas pela sociedade. Para tanto, a interpretação das regras

${ }^{14}$ Cf. HONNETH, Axel. Luta por reconhecimento: a gramática moral dos conflitos sociais. Tradução: Luiz Repa. Apresentação: Marcos Nobre. São Paulo: Editora 34, 2003, 296 p.

${ }^{15}$ Cf. BANKOWSKI, Zenon. Vivendo Plenamente a Lei: a Lei do Amor e o Amor pela Lei. Trad. Lucas Dutra Bertolozzo, Luiz Reimer Rodrigues Rieffel e Arthur Maria Ferreira Neto. Rio de Janeiro, RJ: Elsevier Campus, 2008, 289 p.

${ }^{16}$ Cf. HONNETH, Axel. Luta por reconhecimento: a gramática moral dos conflitos sociais. Tradução: Luiz Repa. Apresentação: Marcos Nobre. São Paulo: Editora 34, 2003, $296 \mathrm{p}$.

${ }_{17}$ Cf. HONNETH, Axel. Luta por reconhecimento: a gramática moral dos conflitos sociais. Tradução: Luiz Repa. Apresentação: Marcos Nobre. São Paulo: Editora 34, 2003, 296 p. 
jurídicas (a moralidade do dever ${ }^{18}$ ) deve incorporar as aspirações ou as promessas, expressas ou implícitas, no processo de elaboração legislativa. A fim de poder se atualizar e conceber as peculiaridades que afetam as sociedades ao longo dos tempos, num processo de mutação contínua, o direito só se constitui pleno de sentido se, de fato, ao perceber a complexidade do real, trouxer à tona a melhor aspiração dos deveres constituídos pela lei ${ }^{19}$. Assim, sendo a lei instrumento necessário à regulação das relações sociais, é imprescindível sua constante avaliação segundo parâmetros empíricos, a fim de que não permaneça intangível nem promova a perpetuação de desigualdades, mas, por outro lado, seja um mecanismo de incentivo ao pleno desenvolvimento do sujeito e seus semelhantes numa comunidade. A aspiração, explicitada por Bankowski ${ }^{20}$, não deve ser concebida como uma mera formalidade para extrair a intenção do legislador ou a vontade da lei, no sentido formal, mas sim como uma estrutura interpretativa dos valores morais imanentes à lei. A aspiração, como elemento de emancipação e de reconhecimento dos sujeitos, deve ser concebida a partir de um ir e vir entre os valores morais universais e a "complexidade do real" 21 .

No sistema jurídico de patentes, há uma promessa (aspiração) de incentivo ao desenvolvimento científico e tecnológico com base no interesse social. A promessa encontra-se tanto no texto constitucional quanto no texto legal de modo explícito. Nesse sentido, vale destacar o dispositivo constante do artigo $5^{\circ}$ da Constituição Brasileira:

XXIX - a lei assegurará aos autores de inventos industriais privilégio temporário para sua utilização, bem como proteção às criações industriais, à propriedade das marcas, aos nomes de empresas e a

${ }^{18}$ Cf. BANKOWSKI, Zenon. Vivendo Plenamente a Lei: a Lei do Amor e o Amor pela Lei. Trad. Lucas Dutra Bertolozzo, Luiz Reimer Rodrigues Rieffel e Arthur Maria Ferreira Neto. Rio de Janeiro, RJ: Elsevier Campus, 2008, 289 p.

${ }^{19}$ Cf. BANKOWSKI, Zenon. Vivendo Plenamente a Lei: a Lei do Amor e o Amor pela Lei. Trad. Lucas Dutra Bertolozzo, Luiz Reimer Rodrigues Rieffel e Arthur Maria Ferreira Neto. Rio de Janeiro, RJ: Elsevier Campus, 2008, 289 p.

${ }^{20}$ Cf. BANKOWSKI, Zenon. Vivendo Plenamente a Lei: a Lei do Amor e o Amor pela Lei. Trad. Lucas Dutra Bertolozzo, Luiz Reimer Rodrigues Rieffel e Arthur Maria Ferreira Neto. Rio de Janeiro, RJ: Elsevier Campus, 2008, 289 p.

${ }^{21}$ BANKOWSKI, Zenon. Vivendo Plenamente a Lei: a Lei do Amor e o Amor pela Lei. Trad. Lucas Dutra Bertolozzo, Luiz Reimer Rodrigues Rieffel e Arthur Maria Ferreira Neto. Rio de Janeiro, RJ: Elsevier Campus, 2008, 289 p. 
outros signos distintivos, tendo em vista o interesse social e o desenvolvimento tecnológico e econômico do País².

No entanto, o equilíbrio entre o interesse social e o desenvolvimento tecnológico e econômico parece estar comprometido pela sobreposição dos interesses do mercado no caso das doenças negligenciadas, como a doença de Chagas. Nesse contexto, é preciso avaliar a efetividade do sistema jurídico de patentes por meio de estudos empíricos os quais revelem, de modo expresso, as incongruências comuns a esse instituto.

\section{Do procedimento metodológico}

A presente pesquisa empírica recorre à análise qualitativa de dados diretos e indiretos para a realização de um processo de inferências. A forma qualitativa de análise empregada neste estudo é a de traços de significação (unobtrusive research), conforme $\mathrm{Babbie}^{23}$. A análise de conteúdo por traços de significação, segundo o autor, inicia-se pela coleta e verificação da medida escolhida, neste caso, os depósitos de pedidos de patentes para a doença de Chagas. Em segundo lugar, a partir do problema e da hipótese de pesquisa, procede-se ao exame dos dados coletados a fim de se alcançar a inferência descritiva. Por fim, a conjugação dos dados e do marco teórico fornecem substrato para formular conclusões acerca da não atratividade do sistema de patentes como provável estrutura de alavancagem no processo de produção inventiva para o combate às doenças negligenciadas, em especial à doença de Chagas.

A natureza dos dados colhidos parte tanto de fontes indiretas, como produção bibliográfica já desenvolvida sobre o tema, quanto de fontes diretas, a exemplo dos dados sobre os depósitos de pedidos de patentes diretamente coletados no site do Instituto Nacional da Propriedade Industrial (INPI) ${ }^{24}$.

\footnotetext{
${ }^{22}$ BRASIL. Constituição da República Federativa do Brasil de 1988. Disponível em: < http://www.planalto.gov.br/ccivil 03/constituicao/constituicaocompilado.htm>.

Acesso em: 10 jun. 2016.

${ }^{23}$ BABBIE, Earl. The Practice of Social Research. Eleventh Edition. Belmont: Thomson Wadsworth, 2007.

24 BRASIL. Instituto Nacional da Propriedade Industrial. Disponível em: $<$ http://www.inpi.gov.br/portal/>. Acesso em: 31 maio 2016.
} 
A etapa da realização de inferências, por fim, é desenvolvida de acordo com o modelo proposto por Epstein e King ${ }^{25}$, de inferências descritivas e causais. Enquanto o pesquisador realiza inferências descritivas partindo de dados conhecidos para atingir fenômenos desconhecidos, a inferência causal é alcançada a partir da compreensão das repercussões de um acontecimento limitado no tempo sobre os fenômenos posteriores ${ }^{26}$.

Os autores afirmam também que uma boa pesquisa empírica é aquela que apresenta aos leitores as informações necessárias para que o trabalho seja replicado ${ }^{27}$. Dessa maneira, no próximo tópico são explicitados o processo de extração dos dados referentes aos depósitos de pedidos de patentes para a construção das tabelas utilizados no estudo.

\section{O manejo e o emprego dos dados coletados}

A escolha da doença de Chagas como alvo do presente estudo foi feita tendo em vista os dados disponíveis em plataformas de saúde e economia, de organizações nacionais e internacionais que atuam no combate à doença, a exemplo dos Médicos Sem Fronteiras e da iniciativa Medicamentos para Doenças Negligenciadas, bem como pelo fato de ser a doença parasitária que mais mata na região da América Latina ${ }^{28}$.

A base de dados de patentes do Instituto Nacional de Propriedade Industrial (INPI) pode ser acessada da seguinte forma: no lado esquerdo da página oficial do INPI ${ }^{29}$ deve-se clicar, respectivamente, no menu

\footnotetext{
${ }^{25}$ EPSTEIN, Lee; KING, Gary. Pesquisa Empírica em Direito: as regras de inferência. São Paulo, SP: Direito GV, 2013. Disponível em: $<$ http://bibliotecadigital.fgv.br/dspace/handle/10438/11444>. Acesso em: 02 jun. 2016.

${ }^{26}$ Cf. EPSTEIN, Lee; KING, Gary. Pesquisa Empírica em Direito: as regras de inferência. São Paulo, SP: Direito GV, 2013, pp. 36-46. Disponível em: $<$ http://bibliotecadigital.fgv.br/dspace/handle/10438/11444>. Acesso em: 02 jun. 2016.

${ }^{27}$ Cf. EPSTEIN, Lee; KING, Gary. Pesquisa Empírica em Direito: as regras de inferência. São Paulo, SP: Direito GV, 2013, pp. 36-46. Disponível em: $<$ http://bibliotecadigital.fgv.br/dspace/handle/10438/11444>. Acesso em: 13 jun. 2018.

${ }^{28}$ INICIATIVA MEDICAMENTOS PARA DOENÇAS NEGLIGENCIADAS (DNDi) (América Latina). <http://www.dndial.org/index.php>. Acesso em: 20 mar. 2016.

29 Cf. BRASIL. Instituto Nacional da Propriedade Industrial. Disponível em: $<$ http://www.inpi.gov.br/portal/>. Acesso em: 31 maio 2016.
} 
"Serviços" - "Patente" - "Busca". Na página posterior ${ }^{30}$, é possível optar por fazer o login ou pesquisar anonimamente a base de dados. Qualquer uma dessas opções redirecionará o usuário para a página "Consulta à Base de Dados do INPI", na qual devem continuar selecionadas as opções "Patente" e "Pesquisa Básica". As caixas de diálogo devem ficar preenchidas da seguinte forma: contenha "todas as palavras", "doença de Chagas", no "resumo". O procedimento foi realizado também trocando a última opção por "título", dada a possibilidade de um depósito possuir o nome da doença somente no título e não no resumo ou o contrário. Para evitar que o mesmo depósito fosse computado duas vezes, foi realizada uma revisão apurada do material coletado, excluindo-se os depósitos eventualmente repetidos.

Os dados extraídos de forma direta das publicações são o número do depósito, a data, o nome do depositante, e o país de origem/prioridade unionista. A primeira tabela criada, denominada "Tabela 1 - Depósitos de pedidos de patentes relacionados à doença de Chagas", abrange, além dos dados acima citados, a coluna "Função", construída com base na leitura do resumo e dos documentos publicados na página do depósito. Tais documentos podem ser visualizados ao clicar em "Documentos publicados" ao final da página.

O período limítrofe de coleta foi convencionado para os depósitos realizados entre 14 de maio de 1996 - data da edição da Lei 9.279/1996, nos termos expostos no artigo 243 - e publicados até 31 de maio de 2016, quando foi realizada a pesquisa na base de dados. Esse recorte temporal forneceu a coleta de um total de 42 publicações de pedidos de depósitos em cujo resumo e título estavam presentes as palavras "doença de Chagas". Como as publicações na plataforma de busca do INPI são atualizadas semanalmente pela Revista de Propriedade Industrial, a revista de número 2369, publicada em 31 de maio de 2016, corresponde à última atualização no banco de dados do INPI antes da construção das tabelas utilizadas nesse artigo.

É preciso esclarecer que quatro depósitos encontrados no período de coleta foram descartados. Os depósitos PI 0714722-8, de 13/07/2007, e PI 9805072-9, realizado em 27/10/1998, foram excluídos em razão da ausência de aplicabilidade direta das invenções à doença de Chagas. A partir da leitura dos documentos disponibilizados para tais depósitos não foi identificada relação com a doença e sim a citação da mesma a título de exemplo. Já os depósitos PP 1100552-1 e PP 1100553-0, ambos realizados em 13/05/1997, foram descartados pela impossibilidade de leitura e análise de suas informações básicas, tendo em vista que suas respectivas

${ }^{30} \mathrm{Cf}$. BRASIL. Instituto Nacional da Propriedade Industrial. Disponível em; $<$ https:/gru.inpi.gov.br/pePI/jsp/patentes/PatenteSearchBasico.jsp>. Acesso em: 13 jun. 2018. 
páginas não estão acessíveis por causa de um erro de serviço da base de dados utilizada no estudo.

A Tabela 1, por sua vez, foi construída a partir da contagem do número de depósito por instituição presente no rol de depositantes. $\mathrm{O}$ objetivo é o de verificar quais agentes estão mais presentes no quadro de depósitos do INPI no que se refere à doença de Chagas, além de averiguar sua natureza, se instituição pública de ensino ou empresa do ramo farmacêutico, por exemplo.

Os documentos que se referem aos depósitos de pedidos de patentes disponíveis na base do INPI apresentam um resumo, um relatório descritivo e as reivindicações da patente a ser concedida. A leitura destes documentos permitiu a classificação da função do objeto a ser patenteado tendo como critérios a prevenção, o diagnóstico e o tratamento da doença de Chagas.

No caso dos depósitos de pedidos de patente, procedeu-se à divisão destes nas seguintes categorias: "Prevenção", categoria de depósitos que visam a evitar a propagação e a contaminação da doença negligenciada; "Diagnóstico", relativo ao método de identificação da doença e; "Tratamento", que se refere à tentativa de combater os sintomas ou curar a doença.

A identificação da função básica do produto foi realizada com o intuito de verificar qual o foco das pesquisas que levaram à sua descoberta e qual a área de atuação para o combate da doença de Chagas, a princípio, seria a mais interessante para os agentes depositantes dos pedidos de patentes. Além disso, também é possível questionar - a partir do perfil do depositante e o foco de suas pesquisas - , se o produto tem potencial para ser colocado no mercado, tendo em vista seus possíveis custos e efetividade frente a outros meios já utilizados para controle da doença.

Antes de apresentar os resultados encontrados durante a pesquisa, é importante frisar que a verificação da concessão ou não do direito de patente, bem como da assiduidade em relação ao pagamento das taxas e anuidades devidas ao INPI, não se encontram no escopo deste estudo. Também é necessário ressaltar novamente que as informações presentes neste estudo se referem a determinado período temporal, compreendido entre 14 de maio de 1996 e 31 de maio de 2016 - data em que os dados foram extraídos da base oficial. Os dados coletados e as respectivas tabelas elaboradas, além das outras informações estudadas, visam, especialmente, à identificação do atual cenário dos depósitos para doença de Chagas, qual o agente dedicado à pesquisa de produtos relacionados à doença e quais seriam estes produtos. 
Esclarece-se, por fim, que a mera permanência dos depósitos de pedidos de patente na base do INPI não configura desídia do órgão em analisar tais processos. Ainda que o pedido tenha sido analisado, informação que pode ser obtida pela leitura dos despachos publicados ao final da página de cada depósito, ele continuará na base oficial para consulta pública.

\section{OS RESULTADOS E O PROCESSO DE INFERÊNCIAS}

\section{As tabelas}

As informações dos depósitos que apresentam "doença de Chagas" no resumo por si só não fariam sentido algum, caso não fossem extraídos e analisados outros dados sobre essa doença no INPI, informações de relatórios e estudos sobre doenças negligenciadas em geral. Desse modo, não seria possível concluir nada além de que foram depositados no INPI, no período limítrofe selecionado, 42 depósitos de pedidos de patente em cujo resumo ou título as palavras "doença de Chagas" estão presentes.

Tabela 1 - Depósitos de pedidos de patentes relacionados à doença de Chagas*

\begin{tabular}{|c|c|c|c|c|c|}
\hline & $\begin{array}{c}\text { № do } \\
\text { depósito }\end{array}$ & $\begin{array}{l}\text { Data do } \\
\text { depósito }\end{array}$ & Função & Depositante & $\begin{array}{l}\text { País de origem } \\
\text { ou Prioridade } \\
\text { Unionista }\end{array}$ \\
\hline 1. & $\begin{array}{c}\text { BR } 102014 \\
0281720\end{array}$ & $11 / 11 / 2014$ & Diagnóstico & $\begin{array}{l}\text { Universidade Federal De } \\
\text { Minas Gerais (BR/MG) }\end{array}$ & Brasil \\
\hline 2. & $\begin{array}{c}\text { BR } 102014 \\
0041079\end{array}$ & $21 / 02 / 2014$ & Diagnóstico & $\begin{array}{l}\text { Universidade Federal De } \\
\text { Minas Gerais (BR/MG) }\end{array}$ & Brasil \\
\hline 3. & $\begin{array}{c}\text { BR } 102013 \\
0319279\end{array}$ & $12 / 12 / 2013$ & Tratamento & $\begin{array}{l}\text { Universidade Estadual } \\
\text { Paulista Júlio de } \\
\text { Mesquita Filho (BR/SP) / } \\
\text { Université De Genève } \\
\text { (CH) }\end{array}$ & Brasil \\
\hline 4. & $\begin{array}{c}\text { BR } 102013 \\
0280968\end{array}$ & $31 / 10 / 2013$ & Tratamento & $\begin{array}{l}\text { Universidade Federal } \\
\text { Fluminense (BR/RJ) }\end{array}$ & Brasil \\
\hline 5. & $\begin{array}{c}\text { BR } 102013 \\
0265586\end{array}$ & $15 / 10 / 2013$ & Tratamento & $\begin{array}{c}\text { Universidade De São } \\
\text { Paulo - USP (BR/SP) / } \\
\text { Universidade Federal De }\end{array}$ & Brasil \\
\hline
\end{tabular}


Tabela 1 - Depósitos de pedidos de patentes relacionados à doença de Chagas*

\begin{tabular}{|c|c|c|c|c|c|}
\hline & $\begin{array}{l}\text { № do } \\
\text { depósito }\end{array}$ & $\begin{array}{l}\text { Data do } \\
\text { depósito }\end{array}$ & Função & Depositante & $\begin{array}{c}\text { País de origem } \\
\text { ou Prioridade } \\
\text { Unionista }\end{array}$ \\
\hline & & & & $\begin{array}{c}\text { Minas Gerais - UFMG } \\
\text { (BR/MG) }\end{array}$ & \\
\hline 6. & $\begin{array}{c}\text { BR } 102013 \\
0173576\end{array}$ & 05/07/2013 & Diagnóstico & $\begin{array}{c}\text { Universidade Federal De } \\
\text { Minas Gerais (BR/MG) / } \\
\text { Fundação De Amparo A } \\
\text { Pesquisa Do Estado De } \\
\text { Minas Gerais - FAPEMIG } \\
\text { (BR/MG) }\end{array}$ & Brasil \\
\hline 7. & $\begin{array}{c}\text { BR } 102013 \\
0021270\end{array}$ & $22 / 01 / 2013$ & Tratamento & $\begin{array}{c}\text { Universidade Federal Da } \\
\text { Paraíba (BR/PB) }\end{array}$ & Brasil \\
\hline 8. & $\begin{array}{l}\text { BR } 102012 \\
0279975\end{array}$ & $31 / 10 / 2012$ & Diagnóstico & $\begin{array}{c}\text { Universidade Federal de } \\
\text { Minas Gerais (BR/MG) / } \\
\text { Fundação de Amparo A } \\
\text { Pesquisa do Estado de } \\
\text { Minas Gerais - Fapemig } \\
\text { (BR/MG) }\end{array}$ & Brasil \\
\hline & $\begin{array}{c}\text { BR } 102012 \\
0190958\end{array}$ & $31 / 07 / 2012$ & Tratamento & $\begin{array}{l}\text { Universidade Federal do } \\
\text { Rio de Janeiro - UFRJ } \\
\text { (BR/RJ) / Universidade } \\
\text { Federal de Alagoas } \\
\text { (BR/AL) }\end{array}$ & Brasil \\
\hline 10. & $\begin{array}{l}\text { BR } 102012 \\
0194287\end{array}$ & $27 / 06 / 2012$ & Tratamento & $\begin{array}{c}\text { Universidade Federal do } \\
\text { Rio Grande do Norte } \\
\text { (BR/RN) }\end{array}$ & Brasil \\
\hline & $\begin{array}{c}\text { BR } 102012 \\
0182149\end{array}$ & $21 / 06 / 2012$ & Prevenção & $\begin{array}{c}\text { Fricontel Comércio De Aço } \\
\text { Inoxidável E Refrigeração } \\
\text { Ltda. (BR/PA) }\end{array}$ & Brasil \\
\hline & $\begin{array}{l}\text { BR } 102012 \\
0103036\end{array}$ & $02 / 05 / 2012$ & Prevenção & $\begin{array}{c}\text { Universidade Federal do Rio } \\
\text { de Janeiro (BR/RJ) }\end{array}$ & Brasil \\
\hline
\end{tabular}


Tabela 1 - Depósitos de pedidos de patentes relacionados à doença de Chagas*

\begin{tabular}{|c|c|c|c|c|}
\hline $\begin{array}{c}\text { № do } \\
\text { depósito }\end{array}$ & $\begin{array}{l}\text { Data do } \\
\text { depósito }\end{array}$ & Função & Depositante & $\begin{array}{c}\text { País de origem } \\
\text { ou Prioridade } \\
\text { Unionista }\end{array}$ \\
\hline 13. $\begin{array}{c}\text { BR } 102012 \\
0018764\end{array}$ & $27 / 01 / 2012$ & Diagnóstico & $\begin{array}{c}\text { Universidade Federal de } \\
\text { Minas Gerais (BR/MG) / } \\
\text { Fundação de Amparo à } \\
\text { Pesquisa do Estado de } \\
\text { Minas Gerais - FAPEMIG } \\
\text { (BR/MG) }\end{array}$ & Brasil \\
\hline 14. PI $1104815-8$ & $31 / 10 / 2011$ & Diagnóstico & $\begin{array}{c}\text { UNIVERSIDADE DE SÃO } \\
\text { PAULO - USP (BR/SP) }\end{array}$ & Brasil \\
\hline 15. PI 1106302-5 & $24 / 10 / 2011$ & Tratamento & $\begin{array}{l}\text { Universidade Federal de } \\
\text { Ouro Preto (BR/MG) / } \\
\text { Fundação de Amparo à } \\
\text { Pesquisa de Estado de MG } \\
\text { (BR/MG) }\end{array}$ & Brasil \\
\hline 16. PI 1103777-6 & $19 / 08 / 2011$ & Tratamento & $\begin{array}{l}\text { Universidade De São Paulo } \\
\text { - USP (BR/SP) / Fundação } \\
\text { De Amparo à Pesquisa Do } \\
\text { Estado De São Paulo - } \\
\text { FAPESP (BR/SP) }\end{array}$ & Brasil \\
\hline 17. PI 0904827-8 & 06/11/2009 & $\begin{array}{l}\text { Prevenção e } \\
\text { diagnóstico }\end{array}$ & $\begin{array}{c}\text { Universidade Federal de } \\
\text { Uberlândia (BR/MG) / } \\
\text { Fundação de Amparo à } \\
\text { Pesquisa do Estado de } \\
\text { Minas Gerais (BR/MG) / } \\
\text { Imunoscan Engenharia } \\
\text { Molecular Ltda-Me } \\
\text { (BR/MG) }\end{array}$ & Brasil \\
\hline 18. PI 0903451-0 & 27/08/2009 & Tratamento & $\begin{array}{c}\text { Universidade Federal do } \\
\text { Piauí (BR/PI) }\end{array}$ & Brasil \\
\hline 19. PI $0805492-4$ & $02 / 12 / 2008$ & Tratamento & $\begin{array}{c}\text { Fundação de Amparo à } \\
\text { Pesquisa do Estado de São } \\
\text { Paulo - FAPESP (BR/SP) / } \\
\text { Universidade de São Paulo - } \\
\text { USP (BR/SP) }\end{array}$ & Brasil \\
\hline 20. PI $0805753-2$ & $31 / 10 / 2008$ & $\begin{array}{l}\text { Prevenção e } \\
\text { tratamento }\end{array}$ & $\begin{array}{c}\text { Carlos de Baeremaecker } \\
\text { Barros (UY) }\end{array}$ & $\begin{array}{c}\text { Argentina } \\
(31 / 10 / 2007)\end{array}$ \\
\hline
\end{tabular}


Tabela 1 - Depósitos de pedidos de patentes relacionados à doença de Chagas*

\begin{tabular}{|c|c|c|c|c|}
\hline $\begin{array}{c}\text { № do } \\
\text { depósito }\end{array}$ & $\begin{array}{l}\text { Data do } \\
\text { depósito }\end{array}$ & Função & Depositante & $\begin{array}{c}\text { País de origem } \\
\text { ou Prioridade } \\
\text { Unionista }\end{array}$ \\
\hline 21. PI 0801906-1 & $15 / 01 / 2008$ & Prevenção & $\begin{array}{l}\text { Universidade Federal de } \\
\text { Minas Gerais (BR/MG) }\end{array}$ & Brasil \\
\hline 22. PI 0806285-4 & $15 / 01 / 2008$ & Prevenção & $\begin{array}{l}\text { Universidade Federal de } \\
\text { Minas Gerais (BR/MG) }\end{array}$ & Brasil \\
\hline 23. PI 0700836-8 & 20/03/2007 & Tratamento & $\begin{array}{c}\text { Fundação Oswaldo Cruz - } \\
\text { FIOCRUZ (BR/RJ) }\end{array}$ & Brasil \\
\hline 24. PI 0709560-0 & $14 / 03 / 2007$ & $\begin{array}{l}\text { Prevenção } \\
\text { e/ou } \\
\text { tratamento }\end{array}$ & $\begin{array}{c}\text { Vib Vzw (BE) / Universiteit } \\
\text { Gent (BE) }\end{array}$ & $\begin{array}{l}\text { Organização } \\
\text { Europeia de } \\
\text { Patentes } \\
(17 / 03 / 2006)\end{array}$ \\
\hline 25. PI 0603872-7 & $24 / 08 / 2006$ & $\begin{array}{l}\text { Diagnóstico e } \\
\text { tratamento }\end{array}$ & $\begin{array}{c}\text { Fundação Universidade de } \\
\text { Brasília (BR/DF) }\end{array}$ & Brasil \\
\hline 26. PI 0603871-9 & $24 / 08 / 2006$ & Tratamento & $\begin{array}{c}\text { Fundação Universidade de } \\
\text { Brasília }(\mathrm{BR} / \mathrm{DF})\end{array}$ & Brasil \\
\hline 27. PI 0502172-3 & $13 / 06 / 2005$ & Tratamento & $\begin{array}{l}\text { Universidade Estadual de } \\
\text { Campinas - UNICAMP } \\
\text { (BR/SP) / Universidade de } \\
\text { São Paulo - USP (BR/SP) }\end{array}$ & Brasil \\
\hline 28. PI 0417231-0 & 06/12/2004 & $\begin{array}{l}\text { Diagnóstico e } \\
\text { tratamento }\end{array}$ & $\begin{array}{l}\text { Mcgill University (CA) / } \\
\text { Vermillion, Inc. (US) }\end{array}$ & $\begin{array}{l}\text { Estados Unidos } \\
\text { (05/12/2003; } \\
\text { 22/04/2004 e } \\
06 / 11 / 2004)\end{array}$ \\
\hline 29. PI 0401107-4 & $13 / 04 / 2004$ & $\begin{array}{l}\text { Prevenção ou } \\
\text { tratamento }\end{array}$ & $\begin{array}{c}\text { Fundação Oswaldo Cruz - } \\
\text { FIOCRUZ (BR) }\end{array}$ & Brasil \\
\hline 30. PI 0310123-1 & $04 / 12 / 2003$ & Diagnóstico & $\begin{array}{c}\text { Louis V. Kirchhoff (US) / } \\
\text { Keiko Otsu (JP) }\end{array}$ & $\begin{array}{l}\text { Estados Unidos } \\
(04 / 12 / 2002)\end{array}$ \\
\hline 31. PI 0305933-2 & $02 / 12 / 2003$ & Prevenção & $\begin{array}{c}\text { Fundação Oswaldo Cruz } \\
\text { (BR/RJ) }\end{array}$ & Brasil \\
\hline
\end{tabular}


Tabela 1 - Depósitos de pedidos de patentes relacionados à doença de Chagas*

\begin{tabular}{|c|c|c|c|c|}
\hline $\begin{array}{c}\text { № do } \\
\text { depósito }\end{array}$ & $\begin{array}{l}\text { Data do } \\
\text { depósito }\end{array}$ & Função & Depositante & $\begin{array}{l}\text { País de origem } \\
\text { ou Prioridade } \\
\text { Unionista }\end{array}$ \\
\hline 32. PI 0315200-6 & $10 / 10 / 2003$ & Diagnóstico & $\begin{array}{l}\text { Osaka Bioscience Institute } \\
\text { (JP) / Japan as Represented } \\
\text { by The Director-General of } \\
\text { National Institute of } \\
\text { Infectious Diseases (JP) }\end{array}$ & Japão (11/10/2002) \\
\hline 33. PI 0211026-1 & $21 / 06 / 2002$ & Tratamento & $\begin{array}{c}\text { Institut Fuer } \\
\text { Pflanzenbiochemie - IPB } \\
\text { (DE) }\end{array}$ & $\begin{array}{l}\text { Alemanha } \\
(21 / 06 / 2001)\end{array}$ \\
\hline 34. PI 0202214-1 & 05/06/2002 & Diagnóstico & $\begin{array}{c}\text { Fundação de Amparo à } \\
\text { Pesquisa do Estado de São } \\
\text { Paulo (BR/SP) }\end{array}$ & Brasil \\
\hline 35. PI 0201237-5 & $25 / 03 / 2002$ & Tratamento & $\begin{array}{l}\text { Fundação de Amparo à } \\
\text { Pesquisa do Estado de São } \\
\text { Paulo - FAPESP (BR/SP) }\end{array}$ & Brasil \\
\hline 36. PI 0206501-0 & $17 / 01 / 2002$ & $\begin{array}{l}\text { Prevenção ou } \\
\text { tratamento }\end{array}$ & $\begin{array}{l}\text { GRÜNENTHAL GMBH } \\
\text { (DE) }\end{array}$ & $\begin{array}{l}\text { Reino Unido } \\
(17 / 01 / 2001) ; \\
\text { Estados Unidos } \\
(13 / 03 / 2001)\end{array}$ \\
\hline 37. PI 0000886-9 & $13 / 01 / 2000$ & Diagnóstico & $\begin{array}{c}\text { Fundação Oswaldo Cruz } \\
\text { (FIOCRUZ) (BR/RJ) / } \\
\text { Fundação Banco do Brasil } \\
\text { (BR/DF) }\end{array}$ & Brasil \\
\hline 38. PI 9903472-7 & 13/08/1999 & Tratamento & $\begin{array}{c}\text { Universidade de São Paulo - } \\
\text { USP (BR/SP) }\end{array}$ & Brasil \\
\hline 39. PI 9903250-3 & 30/07/1999 & Diagnóstico & $\begin{array}{l}\text { FUJIREBIO EUROPE N.V. } \\
\text { (BE) }\end{array}$ & $\begin{array}{l}\text { Organização } \\
\text { Europeia de } \\
\text { Patentes } \\
(30 / 09 / 1998)\end{array}$ \\
\hline 40. PI 9813637-2 & 04/12/1998 & $\begin{array}{l}\text { Prevenção e } \\
\text { diagnóstico }\end{array}$ & Corixa Corporation (US) & $\begin{array}{l}\text { Estados Unidos } \\
(18 / 12 / 1997)\end{array}$ \\
\hline 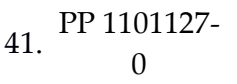 & 14/05/1997 & $\begin{array}{l}\text { Prevenção e } \\
\text { diagnóstico }\end{array}$ & Corixa Corporation (US) & $\begin{array}{l}\text { Estados Unidos } \\
(14 / 02 / 1992)\end{array}$ \\
\hline
\end{tabular}


Tabela 1 - Depósitos de pedidos de patentes relacionados à doença de Chagas*

\begin{tabular}{|c|c|c|c|c|}
\hline $\begin{array}{c}\text { № do } \\
\text { depósito }\end{array}$ & $\begin{array}{l}\text { Data do } \\
\text { depósito }\end{array}$ & Função & Depositante & $\begin{array}{c}\text { País de origem } \\
\text { ou Prioridade } \\
\text { Unionista }\end{array}$ \\
\hline 42. PI 9603267-7 & 02/08/1996 & Diagnóstico & $\begin{array}{c}\text { Universidade Federal de São } \\
\text { Paulo (BR/SP) / Luiz } \\
\text { Rodolpho Raja Gabaglia } \\
\text { Travassos (BR/SP) / Igor } \\
\text { Correia de Almeida (BR/SP) } \\
\text { / Dimas Tadeu Covas } \\
\text { (BR/SP) / Fundação } \\
\text { Hemocentro de Ribeirão } \\
\text { Preto (BR/SP) }\end{array}$ & Brasil \\
\hline
\end{tabular}

Fonte: Os autores a partir do website do INPI

<https://gru.inpi.gov.br/pePI/jsp/patentes/PatenteSearchBasico.jsp>.

*Depósitos realizados a partir de 14/05/1996 e publicados até 31/05/2016 em cujos resumos ou títulos constam "doença de Chagas" e foi verificada relação com a doença.

Data da coleta: $31 / 05 / 2016$

Todavia, ao se atentar para o contexto de desinteresse da indústria farmacêutica no qual se inserem as doenças chamadas negligenciadas ${ }^{31} \mathrm{e}$ coletar no INPI as mesmas informações para outras doenças, é possível afirmar, por exemplo, que uma doença é ainda mais negligenciada que outra. Isso ocorre de fato com a doença de Chagas que, entre dengue, leishmaniose e malária, também pertencentes ao grupo de doenças chamadas de negligenciadas, apresentou a menor quantidade de depósitos: existem disponíveis para consulta, no mesmo período de coleta, 42 depósitos de pedidos de patentes para doença de Chagas diante de 180, 134 e 126 pedidos realizados para as outras doenças, respectivamente. Para efeitos de replicabilidade e em termos de confiabilidade da coleta, foi adotado o mesmo procedimento supracitado, relativo à doença de Chagas, para os casos de dengue, leishmaniose e malária.

Uma das variáveis que pode influenciar os números relacionados à dengue e à malária é a alta mobilidade e a capacidade que seus vetores

31 Cf. SOUZA, Wanderley de (Org.). Doenças Negligenciadas. Rio de Janeiro, RJ: Academia Brasileira de Ciências, 2010. Disponível em: <http://www.abc.org.br/IMG/pdf/doc-199.pdf>. Acesso em: 15 mar. 2016. 
possuem de se proliferarem rapidamente ${ }^{32}$. Com uma zona de infecção maior, o número de pessoas afetadas com a doença tende a ser mais elevado, dando visibilidade a um potencial mercado farmacêutico antes não explorado. Isso, porém, não é regra, pois de acordo com estudo do Instituto de Pesquisa Econômica Aplicada (IPEA), o volume total de gasto com doenças como leishmaniose, malária e tuberculose, representaram $4,6 \%$ da despesa realizada com o programa de AIDS, por exemplo, ainda que o número de ocorrências dessas doenças tenha sido cerca de 3,4 vezes superior ao de pessoas com necessidade de tratamentos antirretrovirais, segundo a mesma pesquisa. Fatores como a capacidade econômica para arcar com os custos do tratamento e o tipo de medicamento a ser desenvolvido também podem ter grande peso quando o assunto é o desenvolvimento de fármacos e a disponibilização dos mesmos para a população acometida pelas doenças negligenciadas ${ }^{33}$.

Tabela 2 - № de depósitos de pedido de patente por depositante(s)

\begin{tabular}{lc}
\hline \multicolumn{1}{c}{ Nome do Depositante } & Quantidade \\
\hline Universidade Federal de Minas Gerais (BR/MG) & 4 \\
Fundação Oswaldo Cruz - FIOCRUZ (BR/RJ) & 3 \\
Universidade Federal de Minas Gerais (BR/MG) / Fundação de Amparo à & 3 \\
Pesquisa do Estado De Minas Gerais - FAPEMIG (BR/MG) & 2 \\
Corixa Corporation (US) & 2 \\
Fundação de Amparo à Pesquisa do Estado de São Paulo (BR/SP) & 2 \\
Fundação Universidade de Brasília (BR/DF) & 2
\end{tabular}

${ }^{32}$ ESCOLA NACIONAL DE SAÚDE PÚBLICA SÉRGIO AROUCA (ENSP). Pesquisador
comenta alerta sobre as doenças transmitidas por vetores. Informe ENSP, 07 abr.
$\begin{array}{llll}2014 . & \text { Disponível } & \text { em: } & <\text { http://www.ensp.fiocruz.br/portal- }\end{array}$ ensp/informe/site/materia/detalhe/35033>. Acesso em: 17 abr. 2016.

${ }^{33}$ BRASIL. Instituto de Pesquisa Econômica Aplicada. Epidemiologia das Doenças Negligenciadas no Brasil e Gastos Federais com Medicamentos. Brasília: 2011, p. 41. Disponível em:

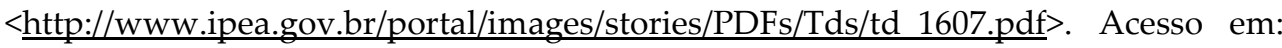
12 abr. 2016. 
Tabela 2 - № de depósitos de pedido de patente por depositante(s)

Nome do Depositante

Quantidade

Universidade de São Paulo - USP (BR/SP) / Fundação de Amparo à

Pesquisa do Estado de São Paulo - FAPESP (BR/SP)

Carlos de Baeremaecker Barros (UY)

Fricontel Comércio De Aço Inoxidável E Refrigeração Ltda. (BR/PA)

FUJIREBIO EUROPE N.V. (BE)

Fundação Oswaldo Cruz (FIOCRUZ) (BR/RJ) / Fundação Banco do Brasil (BR/DF)

GRÜNENTHAL GMBH (DE)

Institut Fuer Pflanzenbiochemie - IPB (DE)

Louis V. Kirchhoff (US) / Keiko Otsu (JP)

Mcgill University (CA) / Vermillion, Inc. (US)

Osaka Bioscience Institute (JP) / Japan as Represented by The Director-

General of National Institute of Infectious Diseases (JP)

Universidade de São Paulo - USP (BR/SP) / Universidade Federal De

Minas Gerais - UFMG (BR/MG)

Universidade Estadual de Campinas - UNICAMP (BR/SP) / Universidade

de São Paulo - USP (BR/SP)

Universidade Estadual Paulista Júlio de Mesquita Filho (BR/SP) /

Université de Genève $(\mathrm{CH})$

Universidade Federal Fluminense (BR/RJ)

Universidade Federal de Ouro Preto (BR/MG) / Fundação de Amparo à

Pesquisa do Estado de MG (BR/MG)

Universidade Federal da Paraíba (BR/PB)

Universidade Federal do Piauí (BR/PI)

1

Universidade Federal do Rio Grande do Norte (BR/RN) 
DOENÇA DE CHAGAS E PATENTES FARMACÊUTICAS

Tabela 2 - № de depósitos de pedido de patente por depositante(s)

\begin{tabular}{|c|c|}
\hline Nome do Depositante & Quantidade \\
\hline Universidade Federal do Rio de Janeiro (BR/RJ) & 1 \\
\hline $\begin{array}{l}\text { Universidade Federal do Rio de Janeiro - UFRJ (BR/RJ) / Universidade } \\
\text { Federal de Alagoas (BR/AL) }\end{array}$ & 1 \\
\hline $\begin{array}{l}\text { Universidade Federal de São Paulo (BR/SP) / Luiz Rodolpho Raja Gabaglia } \\
\text { Travassos (BR/SP) / Igor Correia de Almeida (BR/SP) / Dimas Tadeu Covas } \\
\text { (BR/SP) / Fundação Hemocentro de Ribeirão Preto (BR/SP) }\end{array}$ & 1 \\
\hline $\begin{array}{l}\text { Universidade Federal de Uberlândia (BR/MG) / Fundação de Amparo à } \\
\text { Pesquisa do Estado de Minas Gerais (BR/MG) / Imunoscan Engenharia } \\
\text { Molecular Ltda-Me (BR/MG) }\end{array}$ & 1 \\
\hline Vib Vzw (BE) / Universiteit Gent (BE) & 1 \\
\hline $\begin{array}{l}\text { Fonte: } \quad \text { Os } \quad \text { autores } \\
<\text { https://gru.inpi.gov.br/pePI/jsp/patentes/PatenteSearchBasico.jsp>. }\end{array}$ & INPI \\
\hline
\end{tabular}

Outro indício do desinteresse da indústria farmacêutica privada para com a pesquisa e desenvolvimento de medicamentos para doenças negligenciadas, é o protagonismo das instituições públicas nacionais como depositantes de pedidos de patentes no contexto brasileiro. Entre os 42 depósitos que guardaram relação com a doença, a maioria (31) foi realizada por instituições brasileiras de natureza pública, inseridas nesse número as parcerias feitas, por exemplo, entre fundações estaduais de amparo à pesquisa e universidades públicas.

Tabela 3 - Perfil funcional dos depósitos

\begin{tabular}{cc}
\hline Função & Quantidade \\
\hline Tratamento & 16 \\
Diagnóstico & 12 \\
Prevenção & 5 \\
Prevenção e/ou tratamento & 4 \\
Prevenção e/ou diagnóstico & 3 \\
Diagnóstico e/ou tratamento & 2 \\
\hline
\end{tabular}

Fonte: elaborado pelos autores. 
Por fim, a Tabela 3 demonstra o baixo número de depósitos voltados estritamente para prevenção da doença de Chagas. $\mathrm{O}$ foco na pesquisa e desenvolvimento de produtos voltados ao diagnóstico e tratamento da doença pode estar relacionado à carência de marcadores de diagnóstico mais eficazes, assim como tratamentos menos nocivos ${ }^{34}$. Todavia, o equilíbrio da pesquisa para a seara da prevenção impacta em todo o cenário da doença, na medida em que se trata de um mal que pode ser evitado com melhorias da infraestrutura habitacional, pulverização de inseticidas e utilização de equipamentos de proteção para as pessoas que desenvolvem atividades próximas a áreas florestais ${ }^{35}$. Entretanto, uma limitação que pode ser apontada nesse trabalho se deve ao fato da busca ter sido restrita aos produtos desenvolvidos para doença de Chagas, e não a todo e qualquer tipo de produto que possa interferir no contexto dessa enfermidade sem citá-la diretamente. Os números acima podem estar, relativamente, relacionados com o fato de que escassos são os investimentos em prevenção, o que implica altos gastos com o posterior tratamento da doença. Os maiores gastos anuais com tratamento para doença de Chagas na América Latina, a título de ilustração, foram identificados no Brasil, na média de $\mathrm{R} \$ 129.211 .209^{36}$. Contudo, é preciso desenvolver uma investigação científica mais específica para se comprovar essa correlação entre os números acima e o baixo investimento em prevenção no Brasil, o que foge ao escopo desse trabalho.

\section{Discussão de resultados}

${ }^{34}$ Cf. INICIATIVA MEDICAMENTOS PARA DOENÇAS NEGLIGENCIADAS (DNDi) (América Latina). Relatório Anual DNDi América Latina 2012-2013, p. 29. Disponível em: $<$ http://www.dndial.org/pt/centro-de-documentacao/relatorio-anual.html $>$. Acesso em: 20 mar. 2016.

${ }^{35}$ Cf. ORGANIZAÇÃO MUNDIAL DA SAÚDE (OMS). Trabalhando para Superar o Impacto Global de Doenças Tropicais Negligenciadas: Primeiro relatório da OMS sobre Doenças Tropicais Negligenciadas 2010. Disponível em: $<\underline{\mathrm{http}}$ ://www.who.int/eportuguese/publications/pt/>. Acesso em: 15 mar. 2016.

${ }^{36}$ LEE, Bruce Y. et al.. Global economic burden of Chagas disease: a computational simulation model. The Lancet Infectious Diseases, [s.1.], v. 13, n. 4, p. 342-348, abr. 2013. Disponível em: <http://www.thelancet.com/journals/laninf/article/PIIS1473-3099(13)70002-

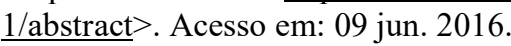


Seguindo as regras de inferência, em Epstein e King ${ }^{37}$, é necessário reconhecer que o estudo de caso único, como os depósitos referidos à doença de Chagas, apresenta uma patente limitação, o que não autoriza realizar generalização mais abrangente acerca das doenças negligenciadas como um todo. Todavia, não se deve desconsiderar o estudo de caso único, como alerta Álvaro Pires ${ }^{38}$. Em vez de se realizar a dedução, cuida-se de promover um processo indutivo de compreensão da complexidade do real. A natureza diagnóstica dessa pesquisa pode servir para se descortinarem novas possibilidades de análise e de compreensão do sistema de patentes não só com referência às doenças negligenciadas mas também com relação a outras possíveis distorções em outros campos da ciência.

A inferência descritiva que se pode alcançar, a partir dos dados e da teoria, se aproxima da hipótese inicial dessa pesquisa, a saber, o atual sistema jurídico de patentes não realiza sua aspiração, de forma global e geral, de promover o desenvolvimento científico atrelado ao interesse social. Se tal não fosse a aspiração do sistema de patentes, por óbvio, o mercado desempenharia a função determinante de selecionar invenções as quais atendessem aos objetivos de lucro e de monopólio das empresas privadas, em geral. Entretanto, está-se lidando com um mecanismo jurídico com o claro objetivo de disseminar a cultura de invenção em todos os campos do saber de forma a atender o interesse social. Diante disso, o estudo do caso de Chagas é demonstrativo dessa falha estrutural do sistema de patentes do ponto de vista de sua coerência lógica interna.

Conforme demonstrado na Tabela 2, os principais agentes que atuam como depositantes de patentes relacionadas à doença de Chagas no INPI são instituições de natureza pública. Esse dado conhecido pode servir para confirmar a tendência de não atratividade do sistema de patentes para alavancar o desenvolvimento de produtos no contexto do setor privado quando se trata de enfermidades referidas à população de baixa renda ou com incidência espacial relativamente limitada, conforme já exposto acima. Além disso, esses dados revelam que o sistema de patentes apresenta relativa atratividade de instituições públicas, porque, em linha de princípio, ao Estado não se deve escusar a preocupação com o reconhecimento de direitos de populações afetadas por doenças negligenciadas. Nesse sentido, os dados demonstram, ao menos parcialmente, uma tendência estatal de promover políticas de incentivo

\footnotetext{
${ }^{37}$ Cf. EPSTEIN, Lee; KING, Gary. Pesquisa Empírica em Direito: as regras de inferência. São Paulo, SP: Direito GV, 2013. Disponível em: $<$ http://bibliotecadigital.fgv.br/dspace/handle/10438/11444> $>$. Acesso em: 02 jun. 2016.

${ }^{38}$ PIRES, Álvaro P.. Amostragem e pesquisa qualitativa: ensaio teórico e metodológico. In: POUPART, Jean et al. A Pesquisa Qualitativa: Enfoques epistemológicos e metodológicos. Petrópolis: Editora Vozes, 2008. p. 154-214. Tradução de: Ana Cristina Arantes Nasser.
} 
ao desenvolvimento de produtos os quais podem suprir necessidades de saúde da população de baixa renda.

Retomando a teoria de Honneth ${ }^{39}$, pode-se apontar que há traços de significação de relativa promoção de direitos e de políticas os quais podem atingir o núcleo do autorrespeito e da estima social na dinâmica do reconhecimento quando se verifica um número bem significativo de entidades públicas destinando recursos para desenvolvimento de produtos ligados à doença de Chagas. Obviamente, é necessário aprofundar a investigação no que se refere ao processo de concessão de auxílios por tais entidades a fim de se comprovar o real propósito dos editais públicos. Afinal, a escolha pelo objeto de estudo pode não ter sido provocada pelo agente público, mas antes derivada da formação individual do pesquisador. No entanto, para aqueles que sustentam o mercado como variável estrutural do sistema jurídico de patentes, é importante demonstrar esse dado, uma vez que um sistema jurídico, cuja promessa consiste no desenvolvimento científico atrelado com o interesse social, padece de suas características mais basais, a saber, a universalidade e a generalidade, quando tal aspiração não se cumpre independentemente dos interesses políticos e privados contingenciais.

$\mathrm{Na}$ Tabela 1, verifica-se um número representativo de depósitos oriundos do estado de Minas Gerais, área endêmica da doença de Chagas no Brasil e que apresenta um número considerável de municípios classificados como de alto risco de transmissão ${ }^{40}$. Esse dado parece confirmar a lógica por trás dos depósitos realizados por instituições públicas, a saber, necessidade de atuação estatal para se corrigir falha institucional no contexto do sistema de patentes para o caso de enfermidades negligenciadas. Entretanto, estudo mais cuidadoso deve ser realizado no sentido de verificar o real propósito dessas políticas institucionais de tais agentes públicos.

Vale destacar, por exemplo, que apenas uma instituição privada nacional efetuou depósito de pedido de patente (depósito $\mathrm{n}^{\mathrm{o}}$ 11) no período analisado. Ademais, ampliando-se a análise para um contexto internacional, também verifica-se o baixo envolvimento de instituições privadas, conforme Tabela 2. Diante disso, os dados brasileiros coletados no INPI apenas servem para confirmar a baixa atratividade do sistema jurídico de patentes quando se está diante de enfermidades

${ }^{39} \mathrm{Cf}$. HONNETH, Axel. Luta por reconhecimento: a gramática moral dos conflitos sociais. Tradução: Luiz Repa. Apresentação: Marcos Nobre. São Paulo: Editora 34, 2003, 296 p.

${ }^{40}$ BRASIL. Fundação Nacional de Saúde. Melhorias Habitacionais para o Controle da Doença de Chagas. Disponível em: <http://www.funasa.gov.br/site/melhorias-habitacionaispara-o-controle-da-doenca-de-chagas/>. Acesso em: 09 jun. 2016. 
negligenciadas, como no caso do mal de Chagas. Isso, entretanto, é indiciário haja vista a necessidade de avaliar a situação de diferentes doenças negligenciadas, como por exemplo, leishmaniose, dengue e malária.

Atentando-se para o argumento mais utilizado pela indústria farmacêutica de que a proteção de suas invenções por meio das patentes consiste num meio para reaver seus investimentos ${ }^{41}$, os dados coletados servem para confirmar que o desinteresse parte do pressuposto econômico, já que as doenças negligenciadas atingem predominantemente populações de baixa renda as quais não representam mercado com capacidade para oferecer retorno a esses laboratórios.

A legislação de patentes não pode ser absorvida por um país sem que haja reflexões profundas sobre as implicações que tais institutos terão na vida em sociedade. Especialmente no que se refere aos países em desenvolvimento, a patente para fármacos deve ser concedida tendo como fundamento o interesse social e atenção aos objetivos das políticas de saúde e bem-estar adotadas pelo Estado. Marcos legislativos, como a Declaração de Doha (2001), incentivam os países a adequarem as leis de propriedade industrial de acordo com suas especificidades, reconhecendo a soberania da proteção à saúde e do acesso a medicamentos sobre o interesse comercial. Isso é se pode inferir do item 17 da Declaração Sobre o Acordo TRIPS e Saúde Pública de $2001^{42}$.

Assim, infere-se que o instituto jurídico da patente, com seus marcos legislativos, não atende às suas aspirações originais, quais sejam: de incentivo ao desenvolvimento tecnológico e econômico aliados, sobretudo, com o interesse social.

Feres e Silva ${ }^{43}$, verificando o desequilíbrio dos índices de novos produtos para as chamadas doenças negligenciadas e outras doenças, também concluem no sentido de que o sistema jurídico de patentes é insuficiente para incentivar a inovação no caso das doenças negligenciadas. Ainda de acordo com os autores ${ }^{44}$, a carga global gerada

${ }^{41}$ ANGELL, Marcia. (2004). The Truth About the Drug Companies. New York: Random House. 303 p.

${ }^{42}$ WORLD TRADE ORGANIZATION (WTO). DOHA WTO MINISTERIAL 2001:

MINISTERIAL DECLARATION. Disponível em:
$<$ https://www.wto.org/english/thewto_e/minist_e $/ \mathrm{min} 01 \_$e/mindecl_e.htm $>$. Acesso em: $12 \mathrm{set}$. 2016.

${ }^{43}$ FERES, Marcos Vinício Chein; SILVA, Alan Rossi. A aspiração do sistema de patentes e o caso dos produtos terapêuticos para doenças negligenciadas. REI - Revista Estudos Institucionais, [s.1.], v. 2, n. 2, p.756-798, 1 fev. 2017. Disponível em: $<$ https://www.estudosinstitucionais.com/REI/article/view/60/115>. Acesso em: 15 jun. 2018.

${ }^{44}$ CHEIN FERES, Marcos Vinício; ROSSI SILVA, Alan. Inovação farmacêutica versus saúde pública: a insuficiência do sistema jurídico de patentes. Revista Direito \& Paz, [S.1.], v. 2, n. 37, p. 228-252, dez. 2017. ISSN 2359-5035. Disponível em: 
por doenças fatais, como as doenças negligenciadas, não se configura como elemento preponderante para a indústria farmacêutica no momento de pesquisar e desenvolver novos produtos para a sociedade.

Constata-se que o cenário da doença de Chagas no que tange aos depósitos de pedidos de patentes no INPI encontra-se literalmente negligenciado. Além disso, cabe destacar que as instituições públicas são, a partir dos dados, fundamentais promotoras do acesso à saúde na realidade da doença de Chagas e das doenças negligenciadas. Os dados revelam, pois, essa preponderância de atuação do poder público nesse setor. Entretanto, é preciso verificar, em novos estudos, se a substituição do poder privado pelo público é, de fato, efetiva para a satisfação das demandas de saúde de tais populações afetadas.

\section{CONSIDERAÇÕES FINAIS}

A pergunta de pesquisa que orientou essa investigação consistiu em averiguar se o sistema jurídico de patentes possui efetividade, no que diz respeito à sua aspiração moral, para o desenvolvimento de produtos de saúde, tendo por foco a análise dos depósitos de patentes para a doença de Chagas. Considerando a relação entre reconhecimento e aspiração do sistema jurídico, a hipótese inicialmente proposta foi a de que há pouca efetividade quando se trata de doenças negligenciadas, não só porque ausente o magnetismo de mercado, mas também porque o mecanismo jurídico de alavancagem não promove seu propósito.

Inicialmente, esperava-se alcançar uma generalização mais abrangente com relação às doenças negligenciadas como um todo. Entretanto, ao longo da coleta e análise dos dados, pode-se verificar que o estudo de caso único, como o é o da doença de Chagas, permitiu apenas um processo indutivo de compreensão da complexidade do real em torno das doenças negligenciadas. Decerto, alcançou-se a inferência descritiva de que, a partir dos dados referentes aos depósitos relativos a doença de Chagas, toma-se conhecimento de uma não atratividade do sistema jurídico de patentes com relação ao incentivo ao desenvolvimento de produtos de saúde voltada para interesses sociais locais no caso das doenças negligenciadas, mais especificamente, a doença de Chagas. Dessa forma, os dados coletados permitiram inferir a existência de falhas com relação à efetividade do sistema quando se trata do cumprimento da aspiração proposta, no caso da doença de Chagas.

$<$ http://www.revista.unisal.br/lo/index.php/direitoepaz/article/view/726>. Acesso em: 15 jun. 2018 . 
Por fim, os dados coletados sugerem outras falhas estruturais no sistema tanto com relação à atuação do poder público de modo mais coerente e efetivo no contexto das doenças negligenciadas quanto com relação às possíveis justificativas pela captura do sistema de patentes pela lógica de mercado. Contudo, investigações mais profundas e mais detalhadas devem ser empreendidas no sentido de dar consistência a estas prováveis hipóteses de pesquisa em futuros trabalhos.

\section{REFERÊNCIAS}

ANGELL, Marcia. (2004). The Truth About the Drug Companies. New York: Random House. 303 p.

BABBIE, Earl. The Practice of Social Research. Eleventh Edition. Belmont: Thomson Wadsworth, 2007.

BANKOWSKI, Zenon. Vivendo Plenamente a Lei: a Lei do Amor e o Amor pela Lei. Trad. Lucas Dutra Bertolozzo, Luiz Reimer Rodrigues Rieffel e Arthur Maria Ferreira Neto. Rio de Janeiro, RJ: Elsevier Campus, 2008, 289 p.

BARBOSA, Denis Borges. Bases Constitucionais da Propriedade Intelectual (2002). 172 p. Disponível em:

$<$ http://denisbarbosa.addr.com/bases4.pdf>. Acesso em: 16 mar. 2016.

Uma Introdução à Propriedade Intelectual. $2^{\mathrm{a}}$ ed. Rio de

Janeiro: Lúmen Júris, 2003.

BRASIL. Constituição da República Federativa do Brasil de 1988.

Disponível em: <

http://www.planalto.gov.br/ccivil_03/constituicao/constituicaocompilad o.htm>. Acesso em: 10 jun. 2016.

Fundação Nacional de Saúde. Melhorias Habitacionais para o

Controle da Doença de Chagas. Disponível em:

$<$ http://www.funasa.gov.br/site/melhorias-habitacionais-para-ocontrole-da-doenca-de-chagas/>. Acesso em: 09 jun. 2016. 
. Instituto Nacional da Propriedade Industrial. Disponível em: < http://www.inpi.gov.br/portal/>. Acesso em: 31 maio 2016. . Instituto de Pesquisa Econômica Aplicada. Epidemiologia das Doenças Negligenciadas no Brasil e Gastos Federais com Medicamentos. Brasília: 2011, 66p. Disponível em: $<$ http://www.ipea.gov.br/portal/images/stories/PDFs/Tds/td_1607.pdf>. Acesso em: 12 abr. 2016.

. Lei no 9.279, de 14 de maio de 1996. Regula direitos e obrigações relativos à propriedade industrial. Disponível em: $<$ http://www.planalto.gov.br/ccivil_03/leis/19279.htm>. Acesso em: 02 abr. 2016.

CHEIN FERES, Marcos Vinício; ROSSI SILVA, Alan. Inovação farmacêutica versus saúde pública: a insuficiência do sistema jurídico de patentes. Revista Direito \& Paz, [S.1.], v. 2, n. 37, p. 228-252, dez. 2017. ISSN 2359-5035. Disponível em:

$<$ http://www.revista.unisal.br/lo/index.php/direitoepaz/article/view/726 >. Acesso em: 15 jun. 2018.

EPSTEIN, Lee; KING, Gary. Pesquisa Empírica em Direito: as regras de inferência. São Paulo, SP: Direito GV, 2013. Disponível em: $<$ http://bibliotecadigital.fgv.br/dspace/handle/10438/11444>. Acesso em: 02 jun. 2016.

ESCOLA NACIONAL DE SAÚDE PÚBLICA SÉRGIO AROUCA (ENSP). Pesquisador comenta alerta sobre as doenças transmitidas por vetores. Informe ENSP, 07 abr. 2014. Disponível em:

$<$ http://www.ensp.fiocruz.br/portalensp/informe/site/materia/detalhe/35033>. Acesso em: 17 abr. 2016.

FERES, Marcos Vinício Chein; SILVA, Alan Rossi. A aspiração do sistema de patentes e o caso dos produtos terapêuticos para doenças 
negligenciadas. REI - Revista Estudos Institucionais, [s.1.], v. 2, n. 2, p.756-798, 1 fev. 2017. Disponível em:

<https://www.estudosinstitucionais.com/REI/article/view/60/115>.

Acesso em: 15 jun. 2018.

FUNDAÇÃO OSWALDO CRUZ (FIOCRUZ) (Invivo). História: Carlos Chagas. Publicado em 07 jan. 2003. Disponível em:

$<$ http://www.invivo.fiocruz.br/cgi/cgilua.exe/sys/start.htm?infoid=109\&s $\mathrm{id}=7>$. Acesso em: 10 jun. 2016.

HONNETH, Axel. Luta por reconhecimento: a gramática moral dos conflitos sociais. Tradução: Luiz Repa. Apresentação: Marcos Nobre. São Paulo: Editora 34, 2003, 296p.

INICIATIVA MEDICAMENTOS PARA DOENÇAS

NEGLIGENCIADAS (DNDi) (América Latina).

<http://www.dndial.org/index.php>. Acesso em: 20 mar. 2016.

Relatório Anual DNDi América Latina 2012-2013. Disponível

em: < http://www.dndial.org/pt/centro-de-documentacao/relatorioanual.html>. Acesso em: 20 mar. 2016.

LEE, Bruce Y. et al.. Global economic burden of Chagas disease: a computational simulation model. The Lancet Infectious Diseases, [s.1.], v. 13, n. 4, p. 342-348, abr. 2013. Disponível em:

$<$ http://www.thelancet.com/journals/laninf/article/PIIS14733099(13)70002-1/abstract>. Acesso em: 09 jun. 2016.

MALAFAIA, Guilherme; RODRIGUES, Aline Sueli de Lima. Centenário do descobrimento da doença de Chagas: desafios e perspectivas. [Editorial]. Revista da Sociedade Brasileira de Medicina Tropical. Uberaba, v. 43, n. 5, p. 483-485, set-out. 2010. Disponível em: < http://www.scielo.br/scielo.php?script=sci_arttext\&pid=S003786822010000500001>. Acesso em: 15 mar. 2016. 


\section{ORGANIZAÇÃO MUNDIAL DA SAÚDE (OMS). Trabalhando para} Superar o Impacto Global de Doenças Tropicais Negligenciadas: Primeiro relatório da OMS sobre Doenças Tropicais Negligenciadas 2010. Disponível em:

<http://www.who.int/eportuguese/publications/pt/>. Acesso em: 15 mar. 2016.

PEDRIQUE, Belen et al. The drug and vaccine landscape for neglected diseases (2000-11): a systematic assessment. The Lancet Glob Health, [s.1.], v. 1, n. 6, p. 371-379, dez. 2013. Disponível em:

<http://www.thelancet.com/journals/langlo/article/PIIS2214109X(13)70148-7>. Acesso em: 20 mar. 2016.

PHILIPSBORN, Peter von. STEINBEIS, Fridolin. BENDER, Max E. REGMI, Sadie Regmi. TINNEMANN, Peter. Poverty-related and neglected diseases - an economic and epidemiological analysis of poverty relatedness and neglect in research and development. Global Health Action, 2015. DOI: <10.3402/gha.v8.25818>. Acesso em: 15 jun. 2018.

PIRES, Álvaro P.. Amostragem e pesquisa qualitativa: ensaio teórico e metodológico. In: POUPART, Jean et al. A Pesquisa Qualitativa: Enfoques epistemológicos e metodológicos. Petrópolis: Editora Vozes, 2008. p. 154-214. Tradução de: Ana Cristina Arantes Nasser.

SOUZA, Wanderley de (Org.). Doenças Negligenciadas. Rio de Janeiro, RJ: Academia Brasileira de Ciências, 2010. Disponível em:

<http://www.abc.org.br/IMG/pdf/doc-199.pdf>. Acesso em: 15 mar. 2016.

VIZZOTTO, Alberto. A função social das patentes sobre medicamentos. São Paulo: LCTE, 2010. 
WORLD HEALTH ORGANIZATION (WHO). Investing to Overcome the Global Impact of Neglected Tropical Diseases: Third WHO Report on Neglected Tropical Diseases 2015. Disponível em:

<http://www.who.int/neglected_diseases/9789241564861/en/>. Acesso em: 15 mar. 2016.

World Health Statitics 2013. Disponível em:

$<$ http://www.who.int/gho/publications/world_health_statistics/EN_WH S2013_Full.pdf?ua=1>. Acesso em: 15 mar. 2016.

WORLD TRADE ORGANIZATION (WTO). DOHA WTO MINISTERIAL 2001: MINISTERIAL DECLARATION. Disponível em: $<$ https://www.wto.org/english/thewto_e/minist_e/min01_e/mindecl_e.ht m>. Acesso em: 12 set. 2016.

DOENÇA DE CHAGAS E PATENTES FARMACÊUTICAS: PESQUISA EXPLORATÓRIA SOBRE A EFETIVIDADE DO SISTEMA DE PATENTES

CHAGAS DISEASE AND PHARMACEUTICAL PATENTS: AN EXPLORATORY RESEARCH ON THE EFFICACY OF THE SYSTEM

Submetido em: 2017- 10- 11 Aceito em: 2018-06-28 